\title{
Estimating abundance of the Endangered onager Equus hemionus onager in Qatruiyeh National Park, Iran
}

\author{
Mahmoud-Reza Hemami and Moslem Momeni
}

\begin{abstract}
Historically, the onager or Persian wild ass Equus hemionus onager, endemic to Iran, was widespread on the arid and semi-arid central steppes but only two natural populations remain. We estimated the population density of the onager in Qatruiyeh National Park using line transect distance sampling. Transects were surveyed on three plains in the Park and the results compared with total counts conducted by the Department of Environment. Our estimate (109 onagers per $100 \mathrm{~km}^{2} ; 95 \%$ confidence interval 67-179) is similar to that obtained by the total counts (137 onagers per $100 \mathrm{~km}^{2}$ ). Distance sampling is therefore a promising method for estimating the abundance of the onager. Using the annual censuses performed by the Department of Environment over the last 13 years we estimated that the population has a growth rate of 0.09 . During the same period the onager population in the Touran Protected Complex has experienced a severe decline. The high density of onagers within the National Park indicates the unsuitability of adjacent habitats, including Bahram-e-Goor Protected Area, for this species. Lack of sufficient security and poorly distributed water sources appear to be the main reasons discouraging onagers from entering the Bahram-e-Goor Protected Area. We recommend and describe management interventions that could potentially assist in maintaining the last surviving onager populations in Iran.
\end{abstract}

Keywords Abundance, Bahram-e-Goor Protected Area, distance sampling, Equus hemionus onager, Iran, onager, Qatruiyeh National Park

\section{Introduction}

$T$ he Asiatic wild ass Equus hemionus, which once ranged 1 from Turkey to northern China and from Kazakhstan to Saudi Arabia and India (Duncan, 1992), is now restricted to protected areas in China, India, Mongolia, Kazakhstan, Turkmenistan and Iran and is categorized as Endangered on the IUCN Red List (Moelhman et al., 2012). The onager

Mahmoud-Reza Hemami (Corresponding author) and Moslem Momeni Department of Natural Resources, Isfahan University of Technology, Isfahan, Iran. E-mail mrhemami@cc.iut.ac.ir

Received 10 September 2010. Revision requested 21 February 2011. Accepted 1 July 2011.
E. hemionus onager, endemic to Iran, is the rarest of the four surviving subspecies.

Numerous manifestations in Persian art and literature attest to the onager's historical abundance throughout the arid and semi-arid steppes of central Iran. Formerly there were four known populations of onager but none have been reported from Kavir National Park and Khosh-Yeilagh Wildlife Refuge since 1986 and 1997, respectively (Hamadanian, 2005). The other populations are in Touran Protected Complex (comprising a national park, wildlife refuge and protected area) in Semnan province, and Bahram-e-Goor Protected Area and Qatruiyeh National Park (formerly Bahram-e-Goor sanctuary zone) in Fars province. The Department of Environment also operates one main captive breeding centre for onagers, in the Kalmand Protected Area in Yazd Province. Habitat destruction and fragmentation, grazing competition with livestock, poaching, poor protection because of an insufficient number of wardens, harassment by feral dogs and severe drought are the most significant threats to onagers (Tatin et al., 2003; Hamadanian, 2005).

Obtaining reliable and unbiased estimates of density or abundance is of importance in understanding population dynamics, and assessing extinction risk and the influence of management activities on the conservation of species. It is therefore a necessary step in any wildlife management project to estimate population size or density. Aerial surveys (Redfern et al., 2002), distance sampling along line transects (Hamrick et al., 2005; Liu et al., 2008; Bardsen \& Fox, 2009), mark-resighting and mark-recapture (McClintock \& White, 2006; Lubow \& Ransom, 2009) and pellet group counts (Haerkoenen \& Heikkilae, 1999; Marques et al., 2001) are some of the most effective methods for estimating ungulate populations. Pellet group counts have been used for abundance estimation of forest-dwelling ungulates (Haerkoenen \& Heikkilae, 1999; Rivero et al., 2004). However, this indirect method requires at least one or two supplementary parameters (faecal pellet disappearance and defecation rate) for converting pellet group density to animal density (Hemami et al., 2005) and has rarely been advocated for ungulates of open country.

Mark-recapture and mark-resighting methods have been successfully employed to estimate ungulate population sizes (e.g. Norton-Griffiths, 1978) but are labour intensive, particularly in large-scale studies. Aerial surveys require high start-up costs and high technical capacity compared to 
other methods and are imprecise relative to effort (GaidetDrapier et al., 2006). Line transect surveys are increasingly used for direct and indirect counts of wild ungulates (e.g. Marques et al., 2001; Hamrick et al., 2005; Waltert et al., 2006; Hemami et al., 2007; Bardsen \& Fox, 2009). This is an appropriate and cost-effective method for estimating the abundance of relatively large and conspicuous species with low population densities over vast open areas (Sutherland et al., 2006) and has been advocated as reliable in several studies of ungulates (Brugière et al., 2005; Valeix et al., 2007).

The lack of knowledge of onager population sizes has been recognized as a hindrance to conservation planning for this taxon (Tatin et al., 2003). The aim of this study was therefore to estimate the abundance of an isolated population of onager in Qatruiyeh National Park, the largest existing population of this taxon, using line transect methodology. We also compared our line transect estimates with the annual total count censuses conducted by the Fars provincial Department of Environment, as the reliability of these censuses has been questioned.

\section{Study area}

The study was conducted in Qatruiyeh National Park, within an area of c. 36,00o ha (Fig. 1). This Park is a semiarid area with vast expanses of plains and some hilly and mountainous terrain. The area was designated as a National Park within Bahram-e-Goor Protected Area in 2008. The mean total annual precipitation is $215 \mathrm{~mm}$ and the mean monthly temperature is $7-37^{\circ} \mathrm{C}$. Zygophyllum eurypterumArtemisia sieberi and A. sieberi are the two dominant vegetation types. Less frequently encountered plant species include Astragalus spp., Stipa barbata and Ephedra spp..
The Kouh-Sorkh Mountain range divides the Park into three plains: Einol-Jalal, Deh-Vazir and Rig-Jamshid. EinolJalal is mainly covered by $A$. sieberi whereas the dominant plant species on the other two plains are Z. eurypterum and A. sieberi. The plains are occupied by onagers and a small population of jebeer gazelle Gazella bennettii. Unlike Bahram-e-Goor Protected Area, the Park has complete protection from hunting, livestock grazing and farming activities. Water sources for wildlife include springs, and water troughs fed by water piped from wells or springs. The permanent spring on Einol-Jalal is the most important water source for onagers in the Park, providing water for artificial troughs in Deh-Vazir. Grey wolves Canis lupus and leopards Panthera pardus occur in the Park, and are potential predators of onagers.

Since 1997 the Fars provincial Department of Environment has carried out systematic censuses to estimate the onager population of Qatruiyeh National Park. The Park is divided into a number of zones and the total count is conducted simultaneously within each zone by separate parties, who remain in radio communication with each other to avoid double counting. According to the results of these censuses the onager population has gradually increased from 93 in 1997 to 285 in 2009.

\section{Methods}

\section{Line transect survey}

Onagers occur throughout the plains of Qatruiyeh National Park and our observations in 2008 to 2009 indicated that onagers rarely enter mountainous areas and steeply-sloped hilly terrains. Therefore, such areas were not considered onager habitat for the purpose of our survey. For the

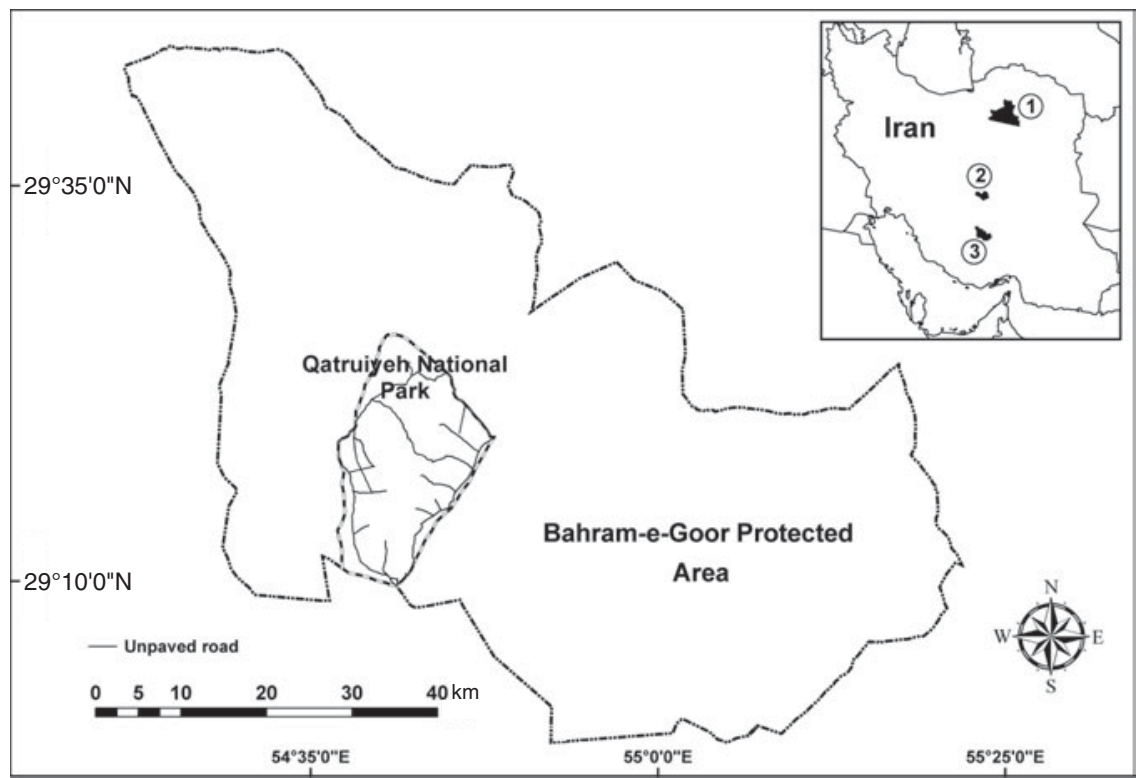

FIG. 1 Bahram-e-Goor Protected Area and Qatruiyeh National Park (3 on the inset) and the distribution of unpaved roads within the Park. The shaded areas on the inset show the current distribution of the onager Equus hemionus onager in Iran (1, Touran Protected Complex; 2, Kalmand Protected Area). 
purpose of our survey we divided onager habitat into the three plains in the Park (Einol-Jalal, Deh-Vazir and RigJamshid). There is a network of unpaved tracks within the Park, used by wardens for patrolling, and we used these to survey a total of $482.2 \mathrm{~km}$ of line transects (including repeated surveys of randomly selected transects). In addition we established a further $46.7 \mathrm{~km}$ of transects randomly within each of the three areas, excluding areas within $1 \mathrm{~km}$ of the tracks. Mean length of transects was $7.14 \pm$ SD $0.59 \mathrm{~km}$.

Visibility of onagers was generally good because of their large size and the flat terrain. Our survey was carried out over 14 days during 15 June to 10 July 2009 by a pair of observers. The weather was sunny and calm during all survey days. Transects were traversed by a motorbike at $20-30 \mathrm{~km} \mathrm{~h}^{-1}$. When a group of onagers was detected the observers stopped and one observer moved towards the original location of the group guided by the second observer signalling with a flag. The geographical coordinates of the detected group were recorded using a global positioning system (GPS). To obtain the perpendicular distance of the detected groups from the transect line the recorded points and transect lines were transferred to a digitized map of the area. The perpendicular distances were then calculated using the NEAR function in ArcGIS v. 9.3 (ESRI, Redlands, USA). Perpendicular distances were not calculated using trigonometry as this would have potentially introduced bias by combining the measurement errors of two separate parameters (i.e. distance from observer to the detected group, and the angle from the transect line to the group). Similarly, Marques et al. (2006) showed that using a GPS is more accurate than using trigonometry.

\section{Analysis of distance data}

Three approaches were used for analysis of distance data: (1) density estimation without stratification of detectability by site, (2) analysis with stratification of detectability by site, and (3) a pooled detection function for the three strata. Data analysis and estimation of density were conducted with DISTANCE V. 5.0 (Thomas et al., 2009).

The frequency distributions of perpendicular distances were used to calculate a probability density function $f(x)$ that models the reduction in detection of onager groups with distance from the transect line. Half-normal, uniform and hazard rate models, with various adjustment terms, were tested in several sets of iterative analyses to select the model giving the best fit to the frequency distribution of the distance data (assessed by a $\chi^{2}$ test of goodness of fit). The final choice of model was that with the lowest value of Akaike's information criterion (AIC).

A truncation distance of $\geq 1,000 \mathrm{~m}$ was chosen, to retain a large proportion of the observations while maximizing model fit, as suggested by Buckland et al., 1993. Observer bias when determining the location of onagers at a distance of $>800 \mathrm{~m}$ could potentially introduce error to the measured perpendicular distances. To reduce this error and to allow detection functions to be fitted whilst maintaining the assumption that the probability of detecting an object on the transect line, $g(0)$, is equal to 1 , distance class intervals were grouped into bands of $200 \mathrm{~m}$.

The effective area surveyed can be obtained by multiplying $\mu$, the effective strip width, by the total length of transects surveyed $(2 L)$. Group density is thus estimated as: $n /(2 \mathrm{~L} \mu)$ where $n$ is the number of detected onager groups within the truncation width of the transect. We also report results for $n / L$, the encounter rate (groups detected per $\mathrm{km}$ of transect). Population density was then estimated by multiplying group density by estimated group size. The overall density obtained based on stratification by plain was estimated using the mean of stratum densities weighted by stratum areas. A regression of observed group size against distance was used to avoid bias when estimating mean group size (Buckland et al., 1993).

In addition to distance sampling the locations of onagers observed during a contemporaneous study of habitat use in the Park were also recorded with a GPS. To estimate abundance it was necessary to exclude areas not commonly used by onagers. To do this we superimposed the locations of these sightings of onagers on a topographical map. Onagers generally avoided sites with a slope of $>10^{\circ}$ although they are occasionally seen in such areas (Mohhammad Hosseini, head of Qatruiyeh National Park, pers. comm.). Areas with slopes $>10^{\circ}$ were therefore excluded from our calculations.

To determine the onager population growth rate $(r)$ we used the equation $N_{t}=N_{\mathrm{o}} e^{r t}$, where $N_{\mathrm{o}}$ is the estimated population size in 1997, $N_{t}$ is the population size at census year $t$, and $e$ is the base of the natural logarithm. The slope of the regression of the natural logarithm of the annual total counts $\left(\log _{e} N\right)$ against census years $(t)$ is $r$ (Sinclair et al., 2006).

\section{Results}

Along a total of $529 \mathrm{~km}$ of transects we detected 734 onagers in 92 groups (Table 1). The highest estimated density of onagers was on the Einol-Jalal plain, followed by Deh-Vazir and Rig-Jamshid. Varying the number of distance class intervals for calculating the detection function affected goodness of fit as well as the precision of some models. For the three plains the best fitting model $(\mathrm{P} \geq 0.60)$ used six distance bands; those with seven, eight or nine bands had poorer fits (Table 2). Density estimation with stratification by plain, using separate detection functions for each, was not precise because we detected too few onager groups on 
TABLE 1 The area of the three sites of potential onager Equus hemionus onager habitat surveyed in Qatruiyeh National Park (Fig. 1), with the number and length of transects traversed (including repeated surveys of randomly selected transects) and numbers of onager groups and individual onagers observed.

\begin{tabular}{llllll}
\hline Site & $\begin{array}{l}\text { Area } \\
(\mathrm{ha})^{2}\end{array}$ & $\begin{array}{l}\text { No. of } \\
\text { transects }\end{array}$ & $\begin{array}{l}\text { Length of } \\
\text { transects }(\mathrm{km})\end{array}$ & $\begin{array}{l}\text { No. of onager } \\
\text { groups observed }\end{array}$ & $\begin{array}{l}\text { No. of onagers } \\
\text { observed }\end{array}$ \\
\hline Rig-Jamshid & 11,129 & 35 & 326 & 34 & 218 \\
Deh-Vazir & 6,492 & 25 & 116 & 29 & 114 \\
Einol-Jalal & 3,173 & 14 & 87 & 29 & 402 \\
Total & 20,794 & 74 & 529 & 92 & 734 \\
\hline
\end{tabular}

each plain. Estimated overall density with and without stratification by plain was similar (Table 2). The most precise estimate resulted from analysis with stratification by plain but using a pooled detection function for the three strata (Table 2).

The mean encounter rate of onagers (Table 2) was $0.23 \pm$ SD $0.12 \mathrm{~km}^{-1}$. We observed group sizes of $2-63$ and solitary onagers comprised 24 of the total of 92 observations (mean group size including solitary onagers $=8.0 \pm \mathrm{SD}$ 11.8). Mean group size after truncation at $1,350 \mathrm{~m}$ was $5.61 \pm \mathrm{SD} 1.46, \mathrm{n}=3$; Table 2). Regression of log group size against detection probability did not reveal any group size bias.

The wide confidence intervals of the three line transect estimates (Table 2) overlap with the estimate obtained by total counts (285) in October 2009. The means of the four estimates are close but the total count is slightly higher. The Department of Environment data show an apparent increase in onager numbers since 1997. We calculated a population growth rate $(r)$ of 0.09 for 1997-2009 (Fig. 2).

\section{Discussion}

Onagers are large, making them easily detectable in open plains except in areas with thick vegetation or gullies and outcrops. As the areas occupied by the onager in Iran are sparsely vegetated and predominantly flat, distance sampling with line transects is a suitable method for estimation of abundance. The constraints of distance sampling include observer bias, weather conditions and the behavioural characteristics of the organism being surveyed (Mahon et al., 1998; Edwards et al., 2000). In the majority of cases onager groups sighted ran away from the observers but we were nevertheless able to note the group's initial location and mark it for subsequent calculation of the perpendicular distance from the transect. We found this method applicable for groups up to 1,000 $\mathrm{m}$ from the transect (105 out of the 111 sighted cases). When analysing by site using separate detection functions for each stratum the highest variation in probability of detection was for Einol-Jalal. The undulating terrain of part of this site may have been responsible for this.
This stratification by plain yielded similar estimates of onager density compared to without stratification but with a poorer fit of detection probability functions because of small sample sizes. Our results suggest that obtaining reliable abundance estimates is feasible without stratification. However, as detection probability and effective strip width were smaller in Einol-Jalal plain it may be advisable to analyse line transect data separately for each plain, although additional survey effort would be required to obtain the desired level of precision.

Of the three plains Eynol-Jalal, which has the highest onager density, also has the highest Artemisia sieberi cover. Results of a habitat selection study using pellet group counts suggested that onagers favour areas with abundant $A$. sieberi cover in the summer (Momeni, 2010). A study in the Touran Protected Complex showed that A. sieberi, Zygophyllum atriplicoides and Peteropyrum aucheri are dominant in habitats recognized as suitable for onagers (Bagheri, 2011).

Our estimate of 109 onagers per $100 \mathrm{~km}^{2}$ (95\% CI 67-179) is much higher than estimates for the kulan Equus hemionus kulan in the Mongolian part of the Gobi desert (4.2-19.1 per $100 \mathrm{~km}^{2}$; Reading et al., 2001). Although we did not detect onagers in the Bahram-e-Goor Protected Area in 41 hours of patrolling, a small proportion of the population may at times occur there (Mohhammad Hosseini, head of Qatruiyeh National Park, pers. comm.). Onagers have also been observed in areas relatively distant from the National Park and the Protected Area. Seasonal movement may change onager population density within the Park but the size of the migratory population is unknown. The mean group size (5.6) observed in this study is much lower than the group size (18.9) reported by Tatin et al. (2003) in the same area in autumn 1997. Mean group sizes of 3.4-35.9 have been reported for kulan in the Gobi desert (Reading et al., 2001).

Our results are consistent with the population counts made by the Fars provincial Department of Environment in October 2009. In our survey two people on a motorcycle required 14 days to survey $529 \mathrm{~km}$ of line transects with distance sampling. The total count performed by the Department of Environment required similar effort (eight people for 2 days using two vehicles). However, there have been occasions when the total count has had to be repeated 


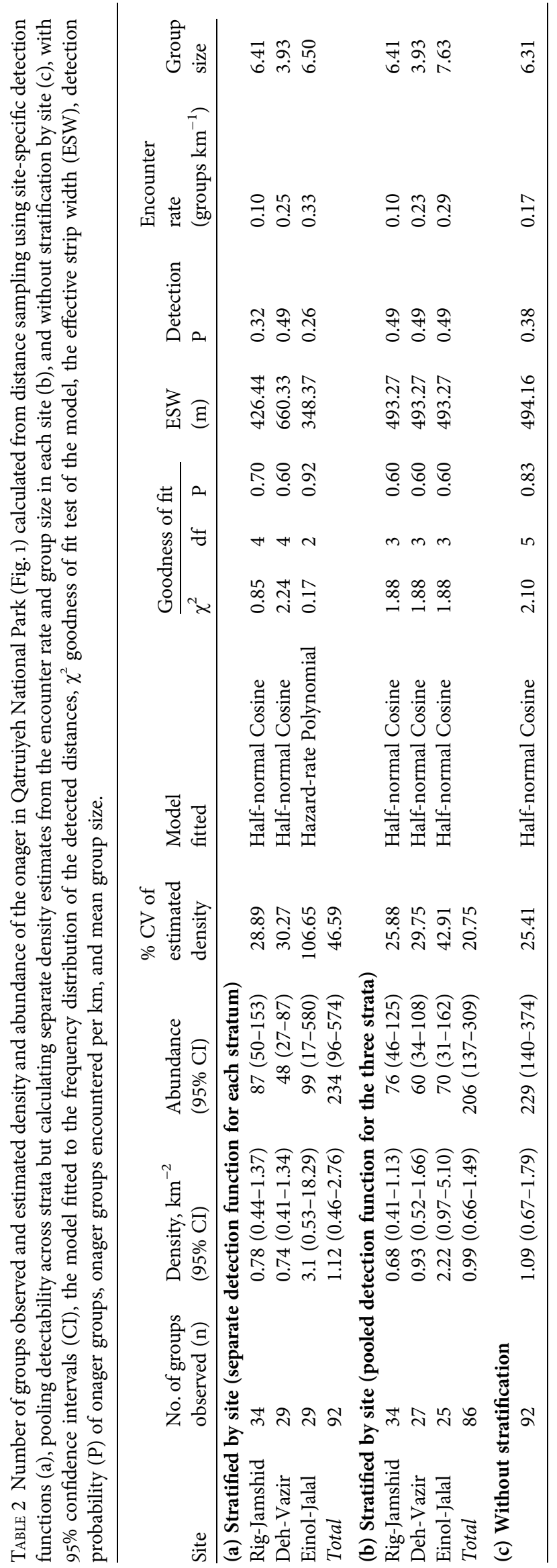

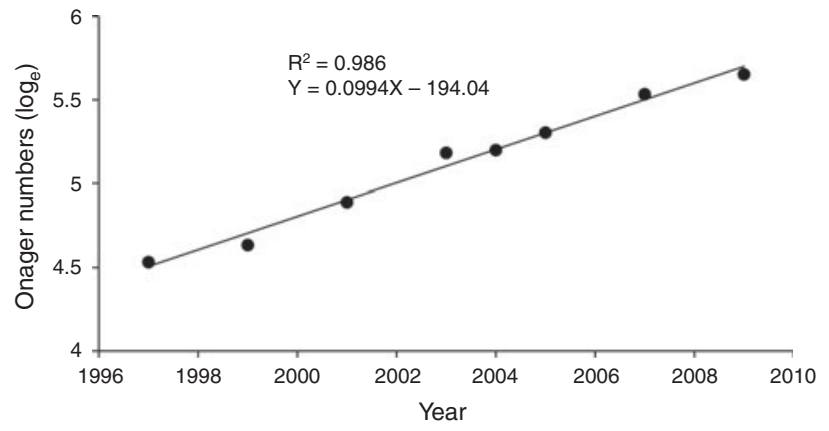

FIG. 2 Linear regression of the natural logarithm of onager numbers for 1997-2009 (data from Fars provincial Department of Environment). The slope of the regression represents the intrinsic annual growth rate $(r)$. Population equation is $N_{t}=93 e^{\text {o.oogr }}\left(R^{2}=0.986, t=20.88, \mathrm{P} \leq 0.001\right)$.

because of unexpected results. Although line transect distance sampling could be a cost-effective method for monitoring onager populations in Iran we recommend that the Department of Environment continue to conduct total counts for a few more years, to facilitate detection of any changes resulting from a change in the census method. Obtaining a reliable population estimate is important, and repeating the count annually with consistency is the key to achieving this goal. Establishing permanent survey transects on the three plains of Qatruiyeh National Park could ensure the consistency of counts and allow comparison of the estimated densities between years and sites.

The onager population in Qatruiyeh National Park has been increasing, with a positive growth rate of 0.09. Annual population growth rates recorded in other studies of E. hemionus are 0.10-0.38 (Moehlman, 2002). Complete protection, exclusion of livestock from the area, increasing the number of water troughs and supplementary feeding of onagers in the dry season may have been the main factors contributing to the positive population trend in the Park.

\section{Management implications}

In addition to the c. 140-374 onagers in Qatruiyeh National Park there are about 150 onagers in Touran Protected Complex (Semnan provincial Department of Environment, unpubl. data). This latter population declined from $660-700$ in 1973 to c. 150 in 2010 (Semnan provincial Department of Environment, unpubl. data). There are also currently a total of 44 onagers (10 males and 34 females) in seven enclosures in Yazd, Kerman, Khorasan-e-jonoobi, Khorasan-e-Razavi and Semnan provinces. Nine of these (three males and six females), escaped from the Tang-e-Hanna enclosure in Kalmand Protected Area and have formed a free-ranging population.

Currently, establishing more captive breeding centres for the onager is the main concern of the Department of Environment. However, all the small founding populations of these centres have originated from two enclosures in 
Kalmand Protected Area, Yazd province. A female-biased sex ratio is also a problem in the captive populations (three captive breeding centres currently hold only a few females and no males).

In non-equilibrium landscapes where spatial and temporal variation in precipitation and productivity of vegetation are high, large-scale movements for foraging are inevitable (Kaczensky et al., 2008). Anecdotal information suggests that movement of onagers has increased in recent years, probably because of the increased population density and drought. Onagers, including dead animals, have reportedly been observed in the north-eastern, southeastern and northern sections of Bahram-e-Goor Protected Area. However, it is not known whether transitory onagers are poached during their migration or return to the Protected Area.

In the Bahram-e-Goor Protected Area seasonal migration, mining activities, disturbance from local roads and visitors, lack of sufficient conservation measures, and insufficient guarding facilities and staff are the main concerns for the conservation of the onager. Poaching occasionally occurs within the Protected Area but the extent is unknown; the cases of poaching detected by the Department of Environment (c. 1 per year over the last 10 years) may be less than the actual figure.

We recommend six management interventions that could potentially assist in maintaining the last surviving onager populations in Iran: (1) Unlike Qatruiyeh National Park most of the onagers in the Touran Protected Complex live outside the National Park, which is part of the Complex. Redrawing the boundary of Touran National Park to include the range of the onager population would provide a more secure habitat. (2) Water availability is a significant factor for habitat selection by onagers (Henley et al., 2007; Kaczensky et al., 2008; Momeni, 2010). Creating permanent artificial water holes would help improve population stability and recovery. Livestock herders need to be prohibited from occupying the present water holes. (3) Genetic management needs to include reinforcement of the captive populations with new individuals. The Department of Environment has already been informed of this need and is currently attempting to capture and transfer some onagers from Bahram-e-Goor Protected Area to the captive breeding centres. (4) As Kaczensky et al. (2008, 2011) suggested, a landscape-level approach is indispensable for conservation management of the onager. We are now initiating satellite tracking studies with the collaboration of the Department of Environment of Iran and the University of Veterinary Medicine of Vienna to clarify the status of the migratory section of the population. Securing migration corridors and important areas adjacent to Qatruiyeh National Park and Bahram-e-Goor Protected Area based on the results of tracking studies may become a conservation priority. (5) An evaluation of the suitability of remaining natural habitats, based on the historical distribution of onagers in Iran, is required, to inform habitat restoration and reintroductions and captive breeding programmes. (6) Ecological education for local people could be beneficial to the success of conservation programmes for the onager. However, until there is an acceptable level of community awareness the number of game guards in the reserves needs to be increased.

\section{Acknowledgements}

We are grateful to the Department of Environment for financial support and permission to work in Qatruiyeh National Park and Bahram-e-Goor Protected Area, all the wardens for their help during fieldwork, Hamid Zohrabi, Hossein Mohammadi and Mohammad Nosrati for their support, Shervin Hess for reviewing the first draft of the paper and two anonymous reviewers for their helpful comments.

\section{References}

BAgheri, M. (2011) Modelling habitat suitability and population density estimation of Equus hemionus onager in Touran Protected Area. MSc thesis, Isfahan University of Technology, Isfahan, Iran.

B ARdsen, B.J. \& Fox, J.L. (2009) Evaluation of line transect sampling for density estimates of chiru Pantholops hodgsoni in the Aru Basin, Tibet. Wildlife Biology, 12, 89-100.

Brugière, D., Dia, M., Diakité, S., Gbansara, M., Mamy, M., Saliou, B. \& Magassouba, B. (2005) Large- and medium-sized ungulates in the Haut Niger National Park, Republic of Guinea: population changes 1997-2002. Oryx, 39, 50-55.

Buckland, S.T., Anderson, D.R., Burnham, K.P. \& LaAke, J.L. (1993) Distance Sampling: Estimating Abundance of Biological Populations. Chapman \& Hall, London, UK.

Duncan, P.B. (1992) Horses and Grasses: The Nutritional Ecology of Equids and their Impact on the Camargue. Springer-Verlag, New York, USA.

Edwards, G.P., de Preu, N.D., Shakeshaft, B.J. \& Crealy, I.V. (2000) An evaluation of two methods of assessing feral cat and dingo abundance in central Australia. Wildlife Research, 27, 143-149.

Gaidet-Drapier, N., Fritz, H., Bourgarel, M., Renaud, P.-C., Poilecot, P. \& Chardonnet, P. (2006) Cost and efficiency of large mammal census techniques: comparison of methods for a participatory approach in a communal area, Zimbabwe. Biodiversity \& Conservation, 13, 735-754.

Haerkoenen, S. \& HeikKilae, R. (1999) Use of pellet group counts in determining density and habitat use of moose Alces alces in Finland. Wildlife Biology, 5, 233-239.

Hamadanian, A. (2005) Onagers (Equus hemionus onager) in Iran, wild and captive. Zoologische Garten, 75, 126-128.

Hamrick, R.G., Pirgalioglu, T., Gunduz, S. \& Carroll, J.P (2005) Feral donkey Equus asinus populations on the Karpaz peninsula, Cyprus. European Journal of Wildlife Research, 51, 108-116.

Hemami, M.R., Watkinson, A.R. \& Dolman, P.M. (2005) Population densities and habitat associations of introduced muntjac Muntiacus reevesi and native roe deer Capreolus capreolus in a lowland pine forest. Forest Ecology and Management, 215, 224-238. 
Hemami, M.R., Watkinson, A.R., Gill, R.M.A. \& Dolman, P.M. (2007) Estimating abundance of introduced Chinese muntjac, Muntiacus reevesi, and native roe deer, Capreolus capreolus, using portable thermal imaging equipment. Mammal Review, 37, 246-254.

Henley, S.R., Ward, D. \& Schmidt, I. (2007) Habitat selection by two desert-adapted ungulates. Journal of Arid Environments, 70, $39-48$.

Kaczensky, P., Ganbaatar, O., von Wehrden, H. \& Walzer, C. (2008) Resource selection by sympatric wild equids in the Mongolian Gobi. Journal of Applied Ecology, 45, 1762-1769.

Kaczensky, P., Kuehn, R., Lhagvasuren, B., Pietsch, S., Yang, W. \& WALZER, C. (2011) Connectivity of the Asiatic wild ass population in the Mongolian Gobi. Biological Conservation, 144, 920-929.

LiU, Z., Wang, X., Teng, L., Cui, D. \& Li, X. (2008) Estimating seasonal density of blue sheep (Pseudois nayaur) in the Helan Mountain region using distance sampling methods. Ecological Research, 23, 393-400.

Lubow, B. \& Ransom, J. (2009) Validating aerial photographic mark-recapture for naturally marked feral horses. Journal of Wildlife Management, 73, 1420-1429.

Mahon, P.S., Banks, P.B. \& Dickman, C.R. (1998) Population indices for wild carnivores: a critical study in sand-dune habitat, south-western Queensland. Wildlife Research, 25, 11-22.

Marques, F.F.C., Buckland, S.T., Goffin, D., Dixon, C.E., Borchers, D.L., Mayle, B.A. \& PeAce, A.J. (2001) Estimating deer abundance from line transect surveys of dung: sika deer in southern Scotland. Journal of Applied Ecology, 38, 349-363.

Marques, T.A., Andersen, M., Christensen-Dalsgatrd, S., Belikov, S., Boltunov, A., Wing, Ø. et al. (2006) The use of global positioning systems to record distances in a helicopter line-transect survey. Wildlife Society Bulletin, 34, 759-763.

McClintock, B.T. \& White, G.C. (2006) Bighorn sheep abundance following a suspected pneumonia epidemic in Rocky Mountain National Park. Journal of Wildlife Management, 71, 183-189.

Moenlman, P.D. (2002) Equids: Zebras, Asses and Horses: Status Survey and Conservation Action Plan. IUCN, Gland, Switzerland, and Cambridge, UK.

Moehlman, P.D., Shah, N. \& Feh, C. (2008) Equus hemionus. In IUCN Red List of Threatened Species v. 2012.1. Http://www. iucnredlist.org [accessed 28 June 2012].

Momeni, M. (2010) Habitat selection and abundance estimation of Persian wild ass Equus hemionus onager in Qatruyieh National Park. MSc thesis, Isfahan University of Technology, Isfahan, Iran.

Norton-Griffiths, M. (1978) Counting Animals. African Wildlife Leadership Foundation, Nairobi, Kenya.
Reading, R.P., Mix, H.M., Lhagvasuren, B., Feh, C., Kane, D.P., Dulamtseren, S. \& Enkhbold, S. (2001) Status and distribution of khulan (Equus hemionus) in Mongolia. Journal of Zoology (London), $258,381-389$.

Redfern, J.V., Viljoen, P.C., Kruger, J.M. \& Getz, W.M. (2002) Biases in estimating population size from an aerial census: a case study in the Kruger National Park, South Africa. South African Journal of Science, 98, 455-461.

Rivero, K., Rumiz, D.I. \& Taber, A.B. (2004) Estimating brocket deer (Mazama gouazoubira and M. americana) abundance by dung pellet counts and other indices in seasonal Chiquitano forest habitats of Santa Cruz, Bolivia. European Journal of Wildlife Research, 50, 161-167.

Sinclair, A.R.E., Fryxell, J.M. \& Caughley, G. (2006) Wildlife Ecology, Conservation, and Management, 2nd edition. Blackwell Publishing, Oxford, UK.

Sutherland, W.J., Greenwood, J.D. \& Robinson, R.A. (2006) Ecological Census Techniques: A Handbook. Cambridge University Press, Cambridge, UK.

Tatin, L., Darreh-Shoori, B.F., Tourend, C., Tatin, D. \& Azmayesh, B. (2003) The last populations of the Critically Endangered onager Equus hemionus onager in Iran: urgent requirements for protection and study. Oryx, 37, 488-491.

Thomas, L., Buckland, S.T., Rexstad, E.A., Laake, J.L., Strindberg, S., Hedley, S.L. et al. (2009) Distance software: design and analysis of distance sampling surveys for estimating population size. Journal of Applied Ecology, 47, 5-14.

Valeix, M., Fritz, H., Dubois, S., Kanengoni, K., Alleaume, S. \& SAID, S. (2007) Vegetation structure and ungulate abundance over a period of increasing elephant abundance in Hwange National Park, Zimbabwe. Journal of Tropical Ecology, 23, 87-93.

Waltert, M., Heber, S., Riedelbauch, S., Lien, J.L. \& Mühlenberg, M. (2006) Estimates of blue duiker (Cephalophus monticola) densities from diurnal and nocturnal line transects in the Korup region, south-western Cameroon. African Journal of Ecology, 44, 290-292.

\section{Biographical sketches}

Mahmoud-Reza Hemami has been cooperating with the Species Survival Commission of IUCN since 1994 and has conducted conservation-related research on various mammalian taxa in Iran and Britain. Moslem Momeni is a wildlife researcher whose main research interests are the ecology and conservation of threatened large herbivores inhabiting arid areas. 